\title{
Detection of circulating tumor cells in non-small cell lung cancer
}

\author{
Gerhard Hamilton, Barbara Rath \\ Society for Research on Biology and Therapy of Cancer, A-1160 Vienna, Austria \\ Correspondence to: Gerhard Hamilton. Society for Research on Biology and Therapy of Cancer, A-1160 Vienna, Austria. \\ Email: Hamilton.SRBTC@gmx.org.
}

Submitted Mar 10, 2016. Accepted for publication Mar 16, 2016.

doi: $10.21037 /$ jtd.2016.03.86

View this article at: http://dx.doi.org/10.21037/jtd.2016.03.86

\section{Treatment of non-small cell lung cancer (NSCLC)}

Lung cancer represents the second most common type of cancer in both men and women in the Western world and is a leading cause of mortality (1). Approximately $80 \%$ of all lung cancers are classed as NSCLC, $15 \%$ are small cell lung cancer (SCLC) and other histological variants account for about $5 \%$ (2). Although NSCLC comprises different histological types, prognosis and therapy for the approximately $40 \%$ adenocarcinomas, $25 \%$ to $30 \%$ squamous cell carcinomas (SCCs) and $10 \%$ to $15 \%$ large cell carcinomas (LCC) is often comparable. For patients with early-stage disease, surgery followed by adjuvant chemotherapy is the optimal treatment (3). However, over $50 \%$ of patients are initially diagnosed with advanced or metastatic disease with worse outcomes. For patients with locally advanced disease, the standard approach is chemoradiotherapy since it offers a small but statistically significant prolongation in survival. For patients with metastatic disease, chemotherapy represents the mainstay of treatment resulting in a median survival of approximately 10 months. The 5 -year survival rate for metastatic disease is a low as $3 \%$, whereas the 5 -year survival rate for all stages is approximately $15 \%$. Therefore, early detection of tumor dissemination and further improvement in diagnostics and treatments are required.

The setting of NSCLC therapies has rapidly been evolving beyond chemotherapy over the last few years. The discovery of oncogenic driver mutations has led to new ways in classifying NSCLC as well as new therapeutic modalities (4). Epidermal growth factor receptor (EGFR) mutations and anaplastic lymphoma kinase (ALK) gene rearrangements were targeted successfully with appropriate tyrosine kinase inhibitors (TKIs). Other driver mutations such as ROS, MET, RET, BRAF have also been handled with specific agents with some success. The administration of inhibitors of cytotoxic T lymphocyte antigen-4 (CTLA-4) and programmed death-1 receptor (PD-1), which prevent tumor-mediated inactivation of the immune system, has led to long-term responses in advanced NSCLC (5). However, personalized treatment directed to driver mutations and immune checkpoint inhibitors in NSCLC is limited to subpopulations of patients.

\section{Relevance of circulating tumor cells (CTCs) in non-small cell lung cancer (NSCLC)}

Practically all tumor types release some malignant cells, termed CTCs, into the circulation. Thus, CTCs represent a subset of tumor cells that have undergone epithelialmesenchymal transition (EMT) and intravasate into the circulatory system or which are shed passively (6). Sampling CTCs may be a viable non-invasive alternative to tissue biopsies for diagnosis and monitoring of lung cancers (7). Otherwise, the only available techniques to monitor disease progression are periodic CT scans done every 3-6 months. Moreover, sequential blood sampling allows to track the dynamic changes of tumor cells during therapy, in particular the development of resistant tumor cell clones. However, in many patients CTCs are quite low in number and need to be enriched from a large population of blood cells (typically 1 CTC per billion blood cells). Nevertheless, CTCs were characterized from patients with early stages of lung cancer (8). Furthermore, CTCs were counted in blood samples of some patients after radical resection for NSCLC and post-surgical presence of CTCs was associated significantly with early disease recurrence (9).

\section{Detection of circulating tumor cells (CTCs)}

The numerous techniques of CTC identification can be split 
into broad groups according to methods of cell enrichment and cell detection (10). The two most commonly applied principles of enrichment are antibody-mediated or physical methods followed by subsequent immunohistochemical enumeration and/or genetic analysis (11). In particular, one approach is anti-epithelial cell adhesion molecule (EpCAM)dependent while the other is marker-independent. The FDA-approved CellSearch ${ }^{\odot}$ technology (Janssen Diagnostics Company, USA) utilizes EpCAM-coated magnetic beads to isolate CTCs from blood of cancer patients. However, CellSearch $^{\odot}$ is approved for metastatic breast, colorectal and prostate cancer but not for lung cancer, where this method exhibits a limited detection efficiency. The markerindependent approaches to isolate CTCs rely on the inherent CTC properties such as size, deformability, or dielectric susceptibility and/or negative selection of white blood cells. Additionally, EpCAM-independent systems allow high throughput and can capture cells subjected to EMT, which have lost epithelial markers. The isolation by size of epithelial tumor cells (ISET) technology is among the earliest developed of the EpCAM-independent approaches (12). According to specific demands, distinct techniques for CTCs may be elected to enumerate, isolate and enrich CTCs for expansion, xenograft implantation or ex vivo drug testing. Newer methods, such as based on binding of specific probes to the folate receptor-1 (FOLR1 or FOL $\alpha$ ) may offer particular advantages.

\section{Characteristics of circulating tumor cells (CTCs) in non- small cell lung cancer (NSCLC)}

Several studies have correlated the presence and the number of CTCs with worse prognosis in lung cancer patients. In early stage lung cancer, the preoperative detection of CTCs is associated with worse disease-free and overall survival. The detection of CTCs in late stage patients and of a high persistent rate of CTCs after chemotherapy is correlated with worse prognosis. CTCs were detected in $80 \%$ of samples from stages IIIA-IV NSCLC patients using ISET compared with $23 \%$ using CellSearch ${ }^{\odot}$ (13). Hofman et al. used ISET technology to isolate CTCs from 208 NSCLC patients with stages I-IV cancer. Fifty percent of these patients had CTCs by morphological examination (14). No direct correlation between numbers of CTCs and disease stage, or other clinicopathological parameters was found. Using the $\mathrm{CellSearch}^{\odot}$ system, there were greater numbers of CTCs in metastatic lung cancer patients compared to patients without distant metastases. In 101 patients with stage III/IV NSCLC, numbers of CTCs were higher in stage IV compared to stage III patients by the CellSearch ${ }^{\odot}$ system. Both the progressionfree survival (PFS: 6.8 vs. 2.4 months) and overall survival (OS: 8.1 vs. 4.3 months) were higher in the favorable group using a threshold of 5 CTCs in $7.5 \mathrm{~mL}$ blood. Reduction in numbers of CTCs with therapy correlated with improved PFS (6.9 vs. 2.4 months) and OS (8.8 vs. 3.9 months). Krebs et al. observed the prevalence of circulating tumor microemboli (CTM) which are highly tumorigenic by ISET technology in $43 \%$ of patients with stage IIIB/IV NSCLC (6). $\mathrm{Nel}$ et al. stained CTCs for both epithelial markers such as EpCAM and pan-cytokeratin (CK) as well as mesenchymal markers such as N-cadherin and CD133 (15). Different subsets of CTC populations were identified which exhibited heterogeneous combinations of epithelial and mesenchymal characteristics. Analysis of CTC markers showed that $40 \%$ of the NSCLC tumors were positive for CK-19, whereas $85 \%$ exhibited both EGFR and MUC-1 (16). Thus, in NSCLC, counting and characterization of CTCs proved their significant role in tumor dissemination and prognosis.

\section{Expression of folate receptor (FOLR1) in cancer}

The FOLR1 is highly expressed on the surface of lung cancer cells but is absent or poorly expressed on the surface of normal blood cells. Member of the FOLR1 family bind folic acid and its reduced derivatives, and transport 5 -methyltetrahydrofolate into cells. Recent estimates suggest that many human carcinomas overexpress FOLR1, including $>90 \%$ of ovarian and endometrial cancers, $>85 \%$ of kidney cancers and approximately $80 \%$ of NSCLCs, among others. Therefore, FOLR1 may be a potential target for capturing CTCs in patients with NSCLC (17). With the advent of targeted therapies directed towards FOLR1, with several such agents in late stage clinical development, the detection of FOLR1 in tissues is of import for patient selection and possibly prognosis and prediction of response. For example, farletuzumab (MORAb-003) is a monoclonal antibody directed to FOLR1, which is currently investigated for the treatment of ovarian cancer (18). Analysis of lung cancer specimens showed that $74 \%$ of adenocarcinomas were positive for FOLR1 expression, whereas only $13 \%$ of SCCs gave positive results (19).

\section{Detection circulating tumor cells (CTCs) by folate receptor (FOLR1)-directed probes}

Fluorescent folate conjugates which bind FOLR1 with 
nanomolar affinity were characterized as tumor-specific ligands in combination with multiphoton fluorescence imaging (20). Intravital flow cytometry noninvasively revealed rare CTCs in vivo as they flow through the peripheral vasculature in experimental animals. The same technique showed a detection limit of approximately two CTCs per $\mathrm{mL}$ in the analysis of whole blood samples from cancer patients, opening chances for early detection of tumor spread. CTCs that were isolated using ApoStream ${ }^{\circledR}$ technology were interrogated to identify FOLR1-positive cells by laser scanning cytometry (21). ApoCell's ApoStream ${ }^{\circledR}$ technology uses dielectrophoresis (DEP) field flow assist a in a microfluidic flow channel to isolate CTCs. Results proved that FOLR1 CTCs can be isolated from patients with metastatic cancers, including NSCLC adenocarcinoma, breast cancer, and ovarian cancer, whereas SSC and normal healthy controls were devoid of FOLR1 CTCs.

A kit to label and detect CTCs through FOLR1 expression in the peripheral blood of cancer patients was developed by Genosaber Biotech (CytoploRare ${ }^{\circledR}$, Shanghai, China). In brief, white blood cells are removed from $3 \mathrm{~mL}$ of blood with anti-CD45 and anti-CD14 immunomagnetic beads followed by labeling of remaining cells with an oligonucleotideFOLR1 probe. Bound ligand-oligonucleotide complexes are stripped from the CTC surface and eventually detected using qPCR. This method was termed ligand-targeted polymerase chain reaction (LT-PCR). The average amount of FOLR1 on tumor cells is $7.5 \times 10^{-18} \mathrm{~mol}$ and the number of lung cancer cells expressing this amount is defined as one CTC unit (CU). Validation of the LT-PCR method for CTC quantification was done with spiked tumor cells (22).

\section{Detection of circulating tumor cells (CTCs) in non-small cell lung cancer (NSCLC) via folate receptor (FOLR1)-LT- PCR: clinical application}

The LT-PCR-FOLR1 method has been investigated for detection of CTCs in NSCLC in several trials (22). The sensitivity of this method was applied to detect rare CTCs in early stage NSCLC patients. The clinical study showed that the initially diagnosed patients have an average of 20.8 CUs with metastatic diseases ( $\mathrm{N}=72), 11.8 \mathrm{CUs}$ with localized diseases $(\mathrm{N}=20)$, and 6.0 CUs with benign diseases $(\mathrm{N}=24)$. With a threshold of 8.5 CUs, the assay could detect $80 \%$ of stage I/II, $67 \%$ of stage III, and $93 \%$ of stage IV cancer. In relation to the control groups, the method could detect cancer with a sensitivity of $82 \%$ and a specificity of $93 \%$, respectively. Thus, LT-PCR would allow enumeration of CTCs in
NSCLC patients at high sensitivity, especially at early stage.

In a similar trial by Yu et al., CTC levels of 153 patients with NSCLC were found to be significantly higher than that of controls (17). With a threshold of 8.64 CUs, the method showed a sensitivity of $73 \%$ and a specificity of $84 \%$ in the diagnosis of NSCLC. In particular, a sensitivity of $67 \%$ was reported in stage I disease. Compared with the common biomarkers such as neuron-specific enolase (NSE), carcinoembryonic antigen (CEA), cancer antigen 125 (CA125), Cyfra21-1 and SSC antigen (SCC Ag), the CTC method showed the highest diagnostic efficiency (area under the receiver operating characteristic; AUC-ROC 0.823).

In a larger study by Chen et al., 756 participants (473 patients with NSCLC, 227 patients with lung benign disease and 56 healthy donors) were included. CTC levels in patients with NSCLC were significantly higher than in those with lung benign disease or healthy donors (23). Compared with CEA, NSE and Cyfra21-1, CTCs displayed the highest AUC-ROC (0.815) in the diagnosis of NSCLC, with a high sensitivity (around 75\%) and specificity (around $85 \%)$. The model combining CTCs with CEA, NSE and Cyfra21-1 was more effective for the diagnosis of NSCLC than the tumor markers alone.

These two studies were corroborated by a study of Wan et al. (24). A total of 50 lung cancer patients served as the NSCLC group, 35 patients with lung benign tumor as the benign group, and 28 healthy subjects as the normal control group. The pathological types included both adenocarcinoma $(\mathrm{n}=25)$ and SSC $(\mathrm{n}=25)$. Here, a CTC amount of $<8.7 \mathrm{CU}$ for LT-PCR-FOLR1 was regarded as negative result. The CTC count was $41 \mathrm{CU}$ in the NSCLC group, $1.03 \mathrm{CU}$ in the benign group and $0.34 \mathrm{CU}$ in the normal group. According to tumor stage, the CTC amount was $5.38 \mathrm{CU}$ in stage I patients, 22.31 CU in stage II patients, $53.39 \mathrm{CU}$ in stage III patients, and $60.14 \mathrm{CU}$ in stage IV patients, respectively. A small number of CTCs were also detected in stage I lung cancer, indicating micrometastasis in the early phases. However, an unresolved issue of this study is the CTC count of approximately $41 \mathrm{CU}$ in both adenocarcinomas and SSC subgroups, despite negative reports on FOLR1 expression in the latter group. In conclusion, these studies have demonstrated the utility of the FOLR1-based LT-PCR in NSCLC and further studies are expected to show the full potential of this technique.

\section{Clinical utility of circulating tumor cells (CTCs) detection tests}

Despite the attractiveness of the enumeration of CTCs as 
liquid biopsy for cancer patients, the respective methods are slow to reach the clinics as routine method (25). To date, there is no consensus on the ideal technical approach, the warranty to get robust and reproducible results and costs of available techniques as well as turnaround time are daunting. For early detection of dissemination and disease monitoring, a relatively simple and preferentially automatable method of CTC counting would be preferable. Actual enrichment and characterization of CTCs itself may be recommended for research purposes and not clinical routine. The method of FOLR1-based LT-PCR seems to possess the potential to constitute a technique of CTC enumeration suitable for application in a clinical setting. Moreover, with a spectrum of appropriate ligands, potentially comprising antibodies, peptides and small chemical molecules, LT-PCR lend itself for extended phenotyping of CTCs in a multiplex, highthroughput fashion.

\section{Acknowledgements}

None.

\section{Footnote}

Conflicts of Interest: The authors have no conflicts of interest to declare.

\section{References}

1. Jemal A, Siegel R, Ward E, et al. Cancer statistics, 2009. CA Cancer J Clin 2009;59:225-49.

2. Yang P, Allen MS, Aubry MC, et al. Clinical features of 5,628 primary lung cancer patients: experience at Mayo Clinic from 1997 to 2003. Chest 2005;128:452-62.

3. Rosell R, Karachaliou N. Lung cancer in 2014: optimizing lung cancer treatment approaches. Nat Rev Clin Oncol 2015;12:75-6.

4. Boolell V, Alamgeer M, Watkins DN, et al. The evolution of therapies in non-small cell lung cancer. Cancers (Basel) 2015;7:1815-46.

5. Bustamante Alvarez JG, González-Cao M, Karachaliou N, et al. Advances in immunotherapy for treatment of lung cancer. Cancer Biol Med 2015;12:209-22.

6. Krebs MG, Metcalf RL, Carter L, et al. Molecular analysis of circulating tumour cells-biology and biomarkers. Nat Rev Clin Oncol 2014;11:129-44.

7. Hanssen A, Loges S, Pantel K, et al. Detection of circulating tumor cells in non-small cell lung cancer. Front
Oncol 2015;5:207.

8. Zhang Z, Shiratsuchi H, Lin J, et al. Expansion of CTCs from early stage lung cancer patients using a microfluidic co-culture model. Oncotarget 2014;5:12383-97.

9. Bayarri-Lara C, Ortega FG, Cueto Ladrón de Guevara A, et al. Circulating tumor cells identify early recurrence in patients with non-small cell lung cancer undergoing radical resection. PLoS One 2016;11:e0148659.

10. Adamczyk LA, Williams H, Frankow A, et al. Current understanding of circulating tumor cells - potential value in malignancies of the central nervous system. Front Neurol 2015;6:174.

11. Ferreira MM, Ramani VC, Jeffrey SS. Circulating tumor cell technologies. Mol Oncol 2016;10:374-94.

12. Vona G, Sabile A, Louha M, et al. Isolation by size of epithelial tumor cells : a new method for the immunomorphological and molecular characterization of circulatingtumor cells. Am J Pathol 2000;156:57-63.

13. Krebs MG, Hou JM, Sloane R, et al. Analysis of circulating tumor cells in patients with non-small cell lung cancer using epithelial marker-dependent and -independent approaches. J Thorac Oncol 2012;7:306-15.

14. Hofman V, Ilie MI, Long E, et al. Detection of circulating tumor cells as a prognostic factor in patients undergoing radical surgery for non-small-cell lung carcinoma: comparison of the efficacy of the CellSearch Assay ${ }^{\mathrm{TM}}$ and the isolation by size of epithelial tumor cell method. Int J Cancer 2011;129:1651-60.

15. Nel I, Jehn U, Gauler T, et al. Individual profiling of circulating tumor cell composition in patients with nonsmall cell lung cancer receiving platinum based treatment. Transl Lung Cancer Res 2014;3:100-6.

16. Warawdekar UM, Sirajuddin MM, Pramesh CS, et al. An approach of selecting appropriate markers from the primary tumor to enable detection of circulating tumor cells in patients with non-small cell lung cancer. J BUON 2015;20:782-90.

17. Yu Y, Chen Z, Dong J, et al. Folate receptor-positive circulating tumor cells as a novel diagnostic biomarker in non-small cell lung cancer. Transl Oncol 2013;6:697-702.

18. Vergote I, Leamon CP. Vintafolide: a novel targeted therapy for the treatment of folate receptor expressing tumors. Ther Adv Med Oncol 2015;7:206-18.

19. O'Shannessy DJ, Yu G, Smale R, et al. Folate receptor alpha expression in lung cancer: diagnostic and prognostic significance. Oncotarget 2012;3:414-25.

20. He W, Wang $\mathrm{H}$, Hartmann LC, et al. In vivo quantitation of rare circulating tumor cells by multiphoton intravital flow 
cytometry. Proc Natl Acad Sci U S A 2007;104:11760-5.

21. O'Shannessy DJ, Davis DW, Anderes K, et al. Isolation of circulating tumor cells from multiple epithelial cancers with ApoStream(®) for detecting (or monitoring) the expression of folate receptor alpha. Biomark Insights 2016;11:7-18.

22. Lou J, Ben S, Yang G, et al. Quantification of rare circulating tumor cells in non-small cell lung cancer by ligand-targeted PCR. PLoS One 2013;8:e80458.

23. Chen X, Zhou F, Li X, et al. Folate receptor-positive circulating tumor cell detected by lt-pcr-based method as

Cite this article as: Hamilton G, Rath B. Detection of circulating tumor cells in non-small cell lung cancer. J Thorac Dis 2016;8(6):1024-1028. doi: 10.21037/jtd.2016.03.86 a diagnostic biomarker for non-small-cell lung cancer. J Thorac Oncol 2015;10:1163-71.

24. Wan JW, Gao MZ, Hu RJ, et al. A preliminary study on the relationship between circulating tumor cells count and clinical features in patients with non-small cell lung cancer. Ann Transl Med 2015;3:352.

25. Ilie M, Hofman V, Long E, et al. Current challenges for detection of circulating tumor cells and cell-free circulating nucleic acids, and their characterization in non-small cell lung carcinoma patients. What is the best blood substrate for personalized medicine? Ann Transl Med 2014;2:107. 